\title{
Genetic drift: the ghost in the genome
}

\author{
Lina Zeldovich
}

Spontaneous mutations can lead scientists down the wrong path, but awareness in academia and new measures by industry are helping to keep genetic drift at bay.

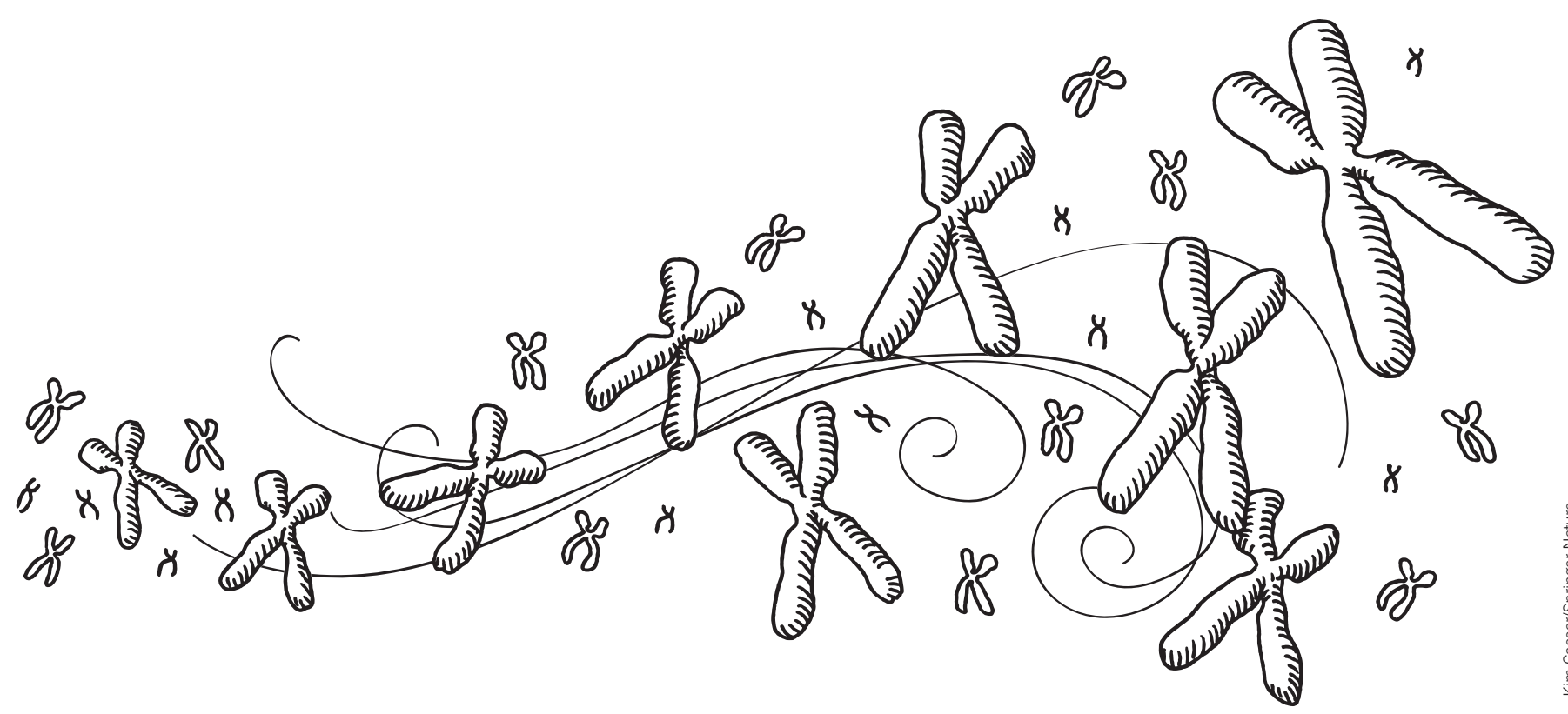

Harvard immunologist Shiv Pillai was puzzled. His team was studying how an enzyme-related malfunction causes B-cells to operate improperly, which contributes to autoimmune diseases. Using knockout mouse models, they had previously traced this malfunction to a mutation in a specific gene, Siae, publishing their findings ${ }^{1}$. But then something strange happened. After Pillai's mice reproduced for 13 more generations, they lost their abnormal B-cell phenotype while still remaining Siaedeficient-thus disproving the previously established connection. Apparently, the phenotype Pillai studied wasn't caused by the genotype he engineered.

To create null-mutations in mice, scientists typically start with embryonic stem cells where a specific gene is targeted for

Lina Zeldovich is an editor and journalist. Correspondence should be addressed to L.Z. (linazeldovich@gmail.com). mutation. The mutated stem cells are then inserted into a blastocyst, which are then implanted into a pseudopregnant female mouse. The resulting mutant offspring are then usually backcrossed into common commercial strains, the most popular being C57BL/6.

But as Pillai's team experienced firsthand, "a rose is a rose is a rose" does not apply to C57BL/6 mouse strains.

Originally developed as a research model by C.C. Little, founder of the Jackson Laboratory, C57BL/ 6 has become the most widely used mouse model in biomedical research, owing mainly to inertia rather than any specific advantages over other mouse types. Over the years, the line has been shared with other institutions/vendors and bred in isolation, generating substrains of C57BL/ 6 with genomes that have drifted over time. While they may look the same, genetic drift can cause significant differences in their phenotypes, especially when scientists go digging into places like their immune systems.

A thorough investigation by Pillai's team, one that involved years of breeding C57BL/6 mice from different vendors, linked the aberrant B-cells' behavior to a completely different culprit: a mutation in a gene named Dock2. As it turns out, the C57BL/6 mice used for their study and those used for further backcrossing afterwards were from different vendors; the Dock2 mutation was only found in mice from the first vendor, indicating the spontaneous mutation most likely arose in their stock.

Pillai was not the only scientist who ran into the Dock 2 conundrum. Deepta Bhattacharya, associate professor of immunology at the Washington University in St. Louis, had also stumbled upon a similar issue with the Dock2 gene. When Bhattacharya was studying mice deficient 
in an irf5 gene, linked to autoimmunity diseases, he attributed the observed immune dysfunctions to that gene. But further investigation proved that this immune dysfunction was related to Dock2 (ref. 2).

These so-called 'quiet mutations' can trick researchers for two reasons, Bhattacharya says. One is that while the Dock2 mutation is a seemingly disadvantaged trait (it causes B-cells to malfunction), it does not have any apparent negative effects on the mice's wellbeing (which might be, in part, because laboratory animals are reared in very favorable conditions.) The second reason is that when researchers observe immune system's aberrant response, they assume it's caused by the gene defect they engineered. "You assume it's because of the gene you intended to mutate," Bhattacharya says. "Not because of some mystery gene you are not aware of."

Genetic consistency and reproducibility In science, the reproducibility of experiments is paramount. It allows independent researchers to prove that previously discovered concepts work, and build upon the established facts. Therefore, for scientists relying on mouse models, it is important that all animals belonging to the same strain have an identical genetic make-up, and thus can perform consistently from one experiment to the next. A scientist using mouse models is not unlike a chemist working with reagents, says Michael Wiles, a senior director of the technology evaluation and development at Jackson Laboratory. While we generally don't think of laboratory mice that way, genetic variations within a mouse strain may skew an experiment's outcome just like the impurity of chemical components can alter the reaction's path or cause the typically safe compounds to explode.

To assure reproducibility, scientists need mice to perform with the consistency of chemical reagents. "So that it would be like a chemist working with sulfur and phosphorus," Wiles says, "each time you did the experiment with these compounds, you would get the same result."

To ensure genetic uniformity, mouse vendors use inbred animals. In a typical protocol, the first two animals, sometimes called Adam and Eve, give rise to the first generation. Their offspring are then used in brother-sister mating for at least 20 generations to force the animals' heterozygous loci to homozygosity, says William Shek, a senior scientific director responsible for research and animal diagnostic services at Charles River Laboratories.

"Very few mammals can withstand inbreeding, but mice can," Wiles says. Within these laboratory-inbred strains, genetic variability is so small that the mice can be almost considered clones. But maintaining genomic consistency over time, when genetic drift can creep in, is still challenging. For researchers trying to reproduce each other's work, the challenge is even greater, given that labs use mice from several different vendors, a small but important fact that goes unmentioned in the methods section of many papers.

\section{Fighting an uphill battle}

All major mouse vendors have in place a set of quality control procedures, checking their mice for genetic uniformity. But according to industry experts, the genetic testing that is currently being done is mainly for genetic contamination, not genetic drift. Because of the large numbers of animals most mouse vendors deal in during a given month or year, the expense and complexity of monitoring genetic drift makes it impractical for any company to pursue.

The only way to fully ensure all animals are genetically identical is by doing a full genome sequence on all of them, but although the cost of this procedure has decreased substantially in recent years, it's still prohibitively expensive. As of today, no affordable technologies exist to reliably check all colonies for genetic drift.

\section{Reset the clock}

Despite not having a clear and affordable way to completely guard against genetic drift, mouse vendors have developed methods to slow it down and decrease the rate that a nuisance genetic mutation will get locked into a colony.

To slow down genetic drift, vendors cryopreserve mouse embryos, using them to replace the foundation colony every so many generations. Taconic Biosciences, Envigo and Charles River do it every 10 generations; Jackson Laboratory every five. The regular replacement of foundation animals from genetically fixed embryos is the only way to effectively slow down genetic drift and prevent genetic changes from accumulating. "That's the only way to avoid this," says Ana Perez, global director of genetic sciences and compliance at Taconic. "You have to go back and reset the clock.”

\section{The benefits of genetic drift}

Although genetic drift can sometimes throw researchers an unexpected curveball, it can also prove to be beneficial to science. For example in 1929, a spontaneous recessive mutation in an inbred colony produced the "shaker1" mice, which exhibited abnormal circling and head-shaking behavior. Over 60 years later, that mutation was linked to a similar one in humans, which causes type 1 Usher syndrome, a genetic disorder characterized by sensory disorders, such as hearing loss. Over the years, other mutations have arisen in various mouse substrains making them useful study models, including some related to schizophrenia, muscular dystrophy, certain types of blindness and other disorders. 
Both researchers and vendors agree that mishaps that skew study resultslike the Dock2 mutation-are relatively rare. When asked whether genetic drift is a source of worry that keeps scientists up at night, Bhattacharya quips, "I sleep OK." "As scientists we know that lot of things we assume are correct, turn out not to be so,"
Pillai says. "So as long as we can rigorously test them, and prove them one way or the other, it's fine." But he hopes that there will come a day when sequencing technologies will permit vendors and laboratories to sequence their animals' genomes on a regular basis to reveal the hidden genetic impurities. In chemistry this would be an equivalent of purifying the reagents before experiments. "I think that is going to happen," Pillai says.

1. Cariappa, A. et al. J. Exp. Med. 206, 125-138 (2009).

2. Purtha, E.W., Swiecki, M., Colonna, M. Diamond, M.S. \& Bhattacharya, D. Proc. Natl. Acad. Sci. USA 109, E898-904. 